\title{
INTRODUCTION TO THE EMF28 STUDY ON SCENARIOS FOR TRANSFORMING THE EUROPEAN ENERGY SYSTEM
}

Europe plays an important role in international climate policy. Among the industrialized countries, European Union Member States have been in favor of relatively stringent emission reductions, consistent with the so-called $2^{\circ} \mathrm{C}$ climate target. In order to achieve the global $2^{\circ} \mathrm{C}$ target, Europe needs to reduce its emissions projected for 2050 significantly, by about $80-95 \%$ relative to 1990 levels. How can such a reduction be achieved? What might cost-effective pathways for achieving this goal look like? Are there different possible transition pathways? How do they compare to the strategy outlined by the European Commission in the "Energy Roadmap 2050"?

This special issue of Climate Change Economics presents the views regarding the aforementioned questions of the EMF28 study of the Stanford Energy Modeling Forum (EMF). EMF employs an international expert platform to improve the understanding of important energy and environmental problems by comparing different modeling approaches and results.

The main objective of the EMF28 study is to provide insights into the debate of the long-term and near-term climate and energy strategies in Europe and address questions related to emission reduction targets, their timing, and technology mix in a way that can help to inform decision-makers in the climate policy arena. In that context, it should be noted that the EU strategy beyond 2020 is not yet defined. In 2011, the EU Commission launched three Roadmaps for the long-term perspective until 2050, based on extensive modeling analysis. One is the "Energy Roadmap 2050", investigating how the long-term energy transformation could take place, which technologies are important, the targets that need to be set, and what the transformation might cost. In addition, in 2013, the EU Commission initiated the debate on policy targets for 2030 with the green paper "A 2030 framework for climate and energy policies". This deals with potential architectures for a post-2020 framework. Contrary to the official Roadmap of the EU Commission which based the analysis of the energy system

This is an Open Access article published by World Scientific Publishing Company. It is distributed under the terms of the Creative Commons Attribution 3.0 (CC-BY) License. Further distribution of this work is permitted, provided the original work is properly cited. 
mainly on the outcome of a single model, the EMF study presents a multi-model perspective and identifies robust and effective strategies based on a suite of thirteen different energy-economy models. The EMF28 study contributes to the inter-model comparison literature on climate policy together with two related studies with an international (EMF27) and US (EMF24) coverage looking at the combined impact of climate policy design and energy technology availability. The model comparison builds on two main types of models: Macroeconomic models that describe the nexus between the economy and the energy system, and partial equilibrium models of the energy system.

The special issue starts with an overview of the EMF28 study (Knopf et al.), discussing mitigation strategies beyond 2020 and identifying robust findings across models in 2030 and 2050. The study shows that despite the existence of several pathways for achieving ambitious climate change mitigation in Europe, the results support the general conclusions of the EU Energy Roadmap. Nearly all the models can achieve the long-term target of reducing GHG emissions by $80 \%$ with GDP costs remaining fairly low for the next decades until 2040. However, in 2050 these costs increase significantly in some models.

De Cian et al. show that the costs also depend considerably on the assumed international policy regime and on the degree of international cooperation. They provide a more focused analysis of the implied alternative European abatement structures and come to the conclusion that more ambitious mitigation action outside Europe could significantly reduce the European burden. This is particularly noticeable when paired with a well-operating global carbon market; technological costs reduce as a result of global learning and $\mathrm{CO}_{2}$ is reduced where it is the cheapest.

A general overview of different types of costs is given by Paltsev and Capros. They discuss, in detail, why the estimates of economic costs of climate policy vary significantly across models. They include a detailed explanation of different cost concepts and how they relate to the different types of models. For EMF28 models there is substantial variation in the estimates of costs, indicating that model comparisons are indeed important in presenting to policy makers the full range of possible costs and benefits.

A more in-depth analysis of mitigation potentials across sectors is undertaken by Förster et al. Their analysis suggests that energy efficiency improvements (including end-use efficiency of economic production and structural change of the economy) are the main cause of declining emissions in the short to medium term. In the long run, decarbonization becomes more important. As mitigation is constrained in the other sectors, the electricity sector has the largest potential for decarbonization.

Another paper by Knopf $e t$ al. explores the interplay between the transformation of the energy system at the European level and that at the level of individual EU Member States. They make the point that in the future the different resource bases and preferences of individual Member States will continue to be reflected in a variety of 
different national energy mixes. Coordination between Member State policies and those at EU level has to be improved to ensure a cost-efficient transformation.

This energy transformation also requires some fundamental changes of the current infrastructure. Holz and von Hirschhausen describe the requirements for the EMF28 scenarios focusing on three major infrastructures: High-voltage electricity transmission lines, natural gas pipelines, and $\mathrm{CO}_{2}$ pipelines. Results suggest that some infrastructure enhancement is required to achieve decarbonization, but that the network development needs can be attained over a reasonable timeframe.

In addition to infrastructure requirements, the need for reliable back-up power production capacities in response to rising renewable energy production has become an important aspect in the debate. Different from other models in EMF28, Schröder et al. find a relatively low level of new non-renewable capacity investments that are required. The main reason is that in their model high electricity prices lead to a substantial decrease in electricity demand.

The EMF28 study could not have been carried out without the commitment of many modeling teams that provided results and their willingness to carefully check on the interpretations and conclusions derived from their models. We are grateful to all modeling teams for their productive cooperation during the entire model comparison exercise. In addition, we would like to thank Elmar Kriegler from the Potsdam-Institute for Climate Impact Research (PIK) for his initiative in pursuing the idea of a European model comparison exercise and Tom van Ierland from DG CLIMA for his feedback and his valuable inputs throughout the project that helped to give policy relevance to our research. We are also grateful to the host organizations of the meetings, namely the Potsdam-Institute for Climate Impact Research (PIK), the Netherlands Environmental Assessment Agency (PBL), and the German Institute for Economic Research (DIW).

John Weyant Stanford University

Brigitte Knopf Potsdam Institute for Climate Impact Research

Enrica De Cian

Fondazione Eni Enrico Mattei and Euro-Mediterranean Center on Climate Change Ilkka Keppo University College London

Detlef P. van Vuuren PBL Netherlands Environmental Assessment Agency and Utrecht University 